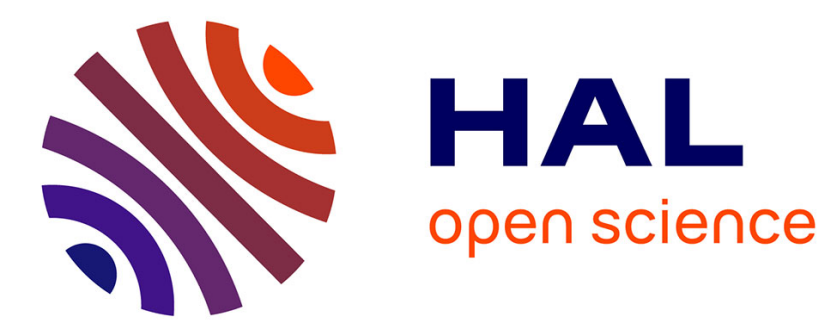

\title{
Une ethnographie visuelle du genre à Medellín. Photographie et pratiques urbaines \\ Camilo Leon-Quijano
}

\section{To cite this version:}

Camilo Leon-Quijano. Une ethnographie visuelle du genre à Medellín. Photographie et pratiques urbaines. Les Annales de la Recherche Urbaine, 2017, 112 (1), pp.116-125. 10.3406/aru.2017.3245 . hal-03505318

\section{HAL Id: hal-03505318 \\ https://hal.science/hal-03505318}

Submitted on 30 Dec 2021

HAL is a multi-disciplinary open access archive for the deposit and dissemination of scientific research documents, whether they are published or not. The documents may come from teaching and research institutions in France or abroad, or from public or private research centers.
L'archive ouverte pluridisciplinaire HAL, est destinée au dépôt et à la diffusion de documents scientifiques de niveau recherche, publiés ou non, émanant des établissements d'enseignement et de recherche français ou étrangers, des laboratoires publics ou privés.

\section{(ㅇ)(1) $\$$}

Distributed under a Creative Commons Attribution - NonCommercial - NoDerivatives $\mid 4.0$ 


\section{Une ethnographie visuelle du genre à Medellín. Photographie et}

\section{pratiques urbaines}

\section{Camilo León-Quijano}

\section{Résumé}

Cet article interroge les modalités de construction du genre dans les pratiques sociospatiales des femmes et des hommes à Medellín (Colombie). En analysant les conditions de réalisation d'une ethnographie visuelle des pratiques urbaines, cet article étudie d'une part les modalités informelles de contrôle genré de l'espace urbain (les transgressions de l'«inattention civile », la modalisation du corps des femmes et les pratiques genrées dans les parcs et les transports). De l'autre, il analyse les usages de la photographie comme outil d'enquête et les potentialités des méthodes participatives de l'image dans une étude sur le genre en ville.

\section{Abstract}

This article analyzes the construction of gender and socio-spatial practices of women and men in Medellín (Colombia). By studying the ways in which the visual fieldwork was realized, this article has two purposes: firstly, it studies the gendered control of urban space through informal interactions (the transgressions of "civil inattention", the modalization of women's bodies and the gendered interactions in parks and transport). Secondly, it explores the use of photography as an investigative tool and the potentials of participatory visual methods in the analysis of gender in urban context.

\section{Citer ce document / Cite this document :}

León-Quijano Camilo. Une ethnographie visuelle du genre à Medellín. Photographie et pratiques urbaines . In: Les Annales de la recherche urbaine, $\mathrm{N}^{\circ} 112,2017$. Le genre urbain. pp. 116-125;

doi : 10.3406/aru.2017.3245

http://www.persee.fr/doc/aru_0180-930x_2017_num_112_1_3245

Document généré le 15/01/2018 


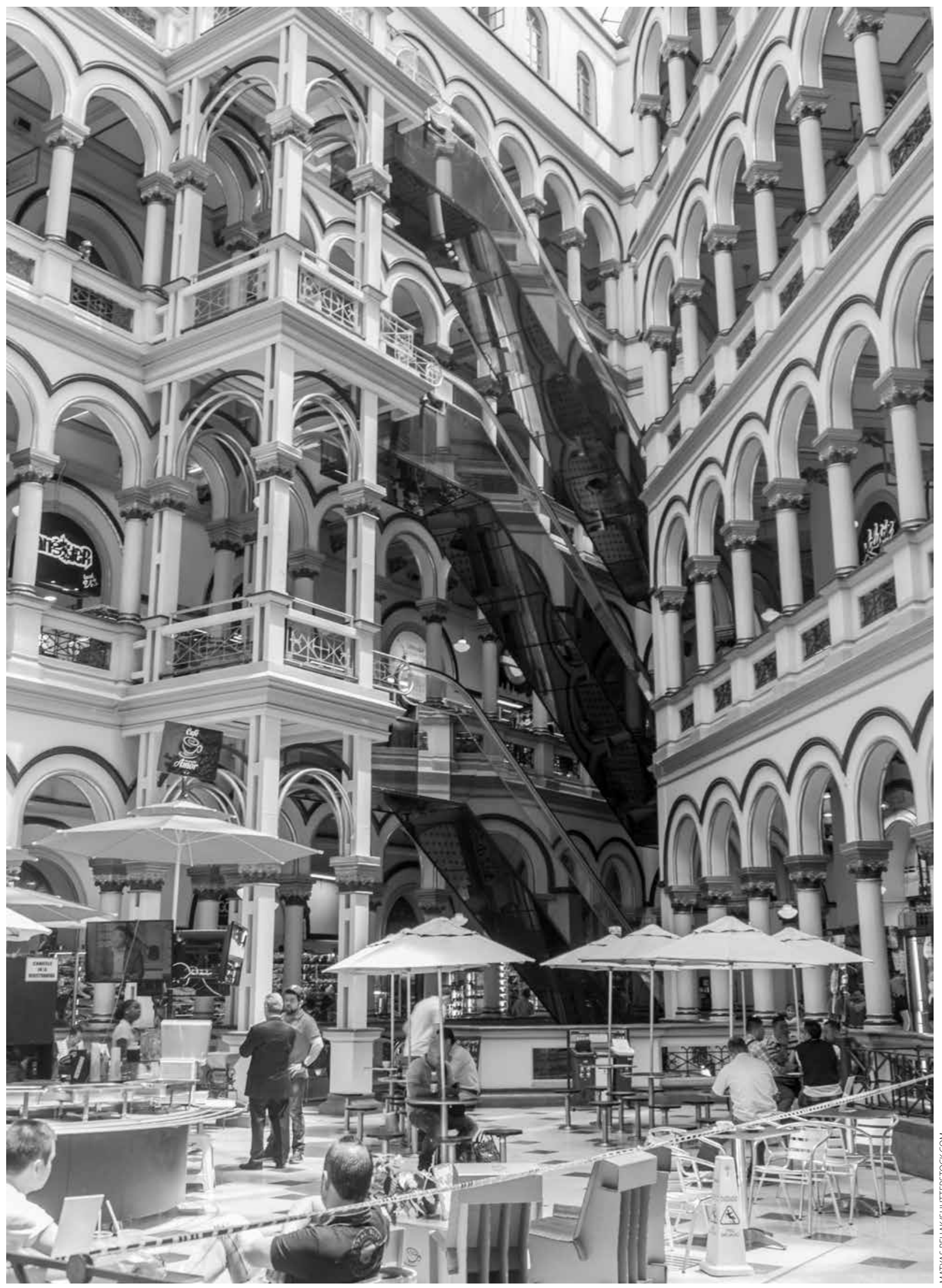




\section{Une ethnographie visuelle du genre à Medellín}

Photographie et pratiques urbaines

\section{Camilo León-Quijano}

Le genre entendu comme "élément constitutif des rapports sociaux fondés sur des différences perçues entre les sexes » (Scott, 1988, p. 14l) permet d'analyser de manière relationnelle les rapports de pouvoir entre femmes et hommes dans la ville'. Si à Medellín la violence est un dispositif formel (direct) de contrôle sociospatial genré, d'autres types d'interactions - moins agressives - agissent comme mode de contrôle non directement violent des pratiques urbaines des acteurs. Nous avons ainsi mené une recherche en utilisant la photographie comme outil d'enquête ethnographique pour décrire et interpréter les pratiques urbaines genrées des femmes et des hommes dans certains quartiers de la ville.

En privilégiant l'approche goffmanienne (Goffman, $1977^{3}$ ), nous avons consacré une partie de notre enquête à

1. Ce travail a été réalisé dans le cadre du mémoire de master intitulé Nosotras mismas les hemos dado ese poder (Construction du genre dans l'espace urbain : une socio-ethnographie visuelle des pratiques urbaines à Medellín et à Sarcelles), soutenu en 2015 à l'EHESS. L'enquête ethnographique a été mené à Medellín entre 2014 et 2015. Cet article reprend des extraits du deuxième et troisième chapitre.

2. La violence en sens large (sexuelle, physique, domestique...).

3. L'article d'Erving Goffman publié dans l'ouvrage L'arrangement des sexes (1977) traite indirectement du genre dans l'espace urbain. Carol B. Gardner, ancienne doctorante de Goffman, analyse de manière plus poussée cette réflexion. L'interaction verbale, les gestes, les conventions, la forme physique de s'exprimer, font partie d'un ensemble de conduites humaines qui se dégagent au moment des interactions. Ils garantissent à la fois l'ordre rituel et la préservation de la face, but ultime des interactions entre individus (Goffman, 1963, pp. 5-45). Pour Gardner, l'analyse des interactions dans l'espace public ne peut pas être entendue comme " gender neutral » (Gardner, 1989, p. 43). Selon elle, il ne faudrait pas analyser les comportements des femmes dans l'espace public, mais « déconstruire » l'espace public en soi. Dans ce cadre, cette enquête étudie les modalités de catégorisation et de construction genré de l'espace. En analysant les modalités d'engagement et d'arrangement entre les sexes, cet article vise à décrire le rôle du genre dans la construction de l'ordre interactionnel en ville. l'étude du rôle des interactions informelles dans la catégorisation genrée des pratiques sociospatiales : les piropos ${ }^{4}$, les insultes, les regards, les engagements oraux anonymes dans la rue, et plus largement les « transgressions de l'inattention civile» (Goffman, 1963, p. 84).

Nous avons privilégié l'usage de la photographie comme outil heuristique pour éclairer une réalité visuelle constituante et constitutive de l'expérience genrée de l'espace urbain. Entre 2014 et 2015 nous avons mené vingtsix entretiens approfondis dans plusieurs quartiers de la ville $^{6}$. En reprenant les techniques d'interview photographique $^{7}$ (Collier et Collier, 1986 ; Harper, 2002), nous

4. Un piropo est un « commentaire de rue » qui vise à être perçu comme un compliment. Il est fait par un homme (à une femme) dans la rue.

5. Un ensemble hétérogène et relativement récent de techniques visuelles qu'utilise l'image pour « impliquer » les enquêté-e-s dans un processus de recherche d'action participative. Au cours des trente dernières années, ces techniques se sont développées dans l'esprit de " rééquilibrer » les écarts de pouvoir entre l'enquêteur et l'enquêté-e, notamment dans le monde anglophone (cf. Pink, 2013).

6. Notamment dans les Comuna 1, 2, 8, 10, 13, 14, 15, 16.

7. Ou photo-elicitation, cf. Harper, 2002. Nous empruntons le terme elicitation à la sociologie visuelle américaine, notamment à Douglas Harper (2002), qui évoque les multiples usages de la photographie dans le cadre d'un entretien. Ce dernier parle de photo-elicitation à ce propos (proche de l'« entretien photographique » de Collier et Collier, 1986). Nous employons la notion d'elicitation (soustraire des informations) pour désigner le travail de construction collective de sens, pour analyser l'expérience vécue à l'aide de la photographie. En confrontant le récit visuel et verbal des enquêté-e-s, nous « élicitons » à la fois des savoirs et des informations. La communication des expériences urbaines et l'interprétation de cette expérience passe par un support non verbal. Les images deviennent ainsi des outils de description et permettent d'interpréter collectivement l'expérience sociospatiale.

Les Annales de la recherche urbaine $n^{\circ} 110,2015$, pp. 116-125, (C)MEEM, Puca. 
Photographies illustratives des ateliers réalisés à Medellín entre 2014 et 2015

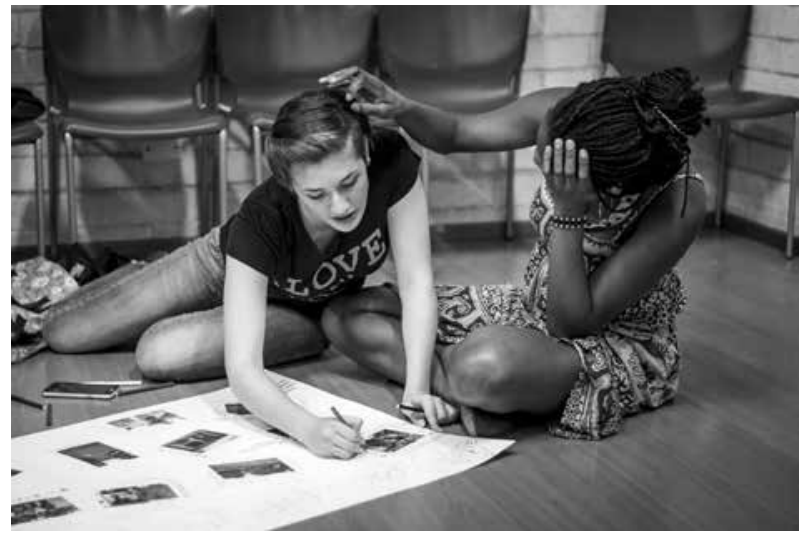

Création des cartographies visuelles de genre (CVG) à Belén, Medellín.

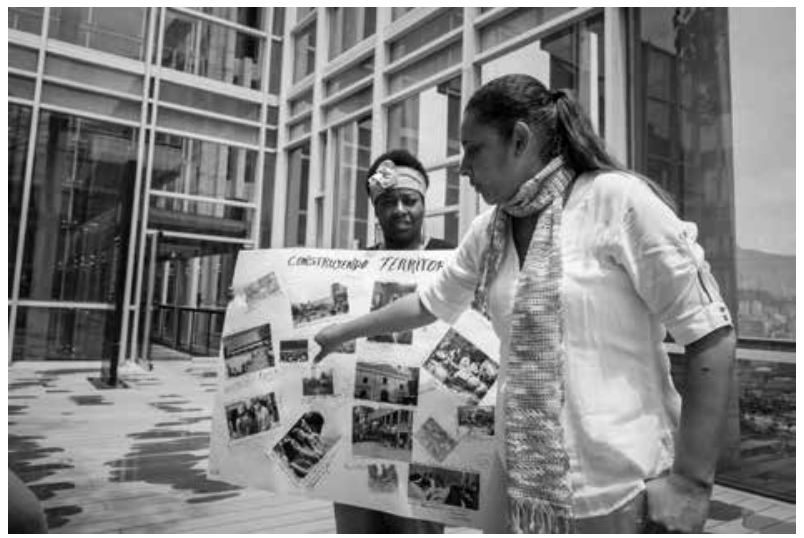

Socialisation collective des cartographies visuelles de genre à la Mairie de Medellín

avons utilisé la photographie comme un moyen pour décrire, interpréter et partager l'expérience sociospatiale des enquêté-e-s au cours des entretiens ${ }^{8}$.

Nous avons donc animé dix-neuf ateliers photo-ethnographiques avec cinquante-deux enquêté-e-s dans plusieurs quartiers de la ville. L'idée était d'explorer les formes d'interaction et de catégorisation genrée des pratiques sociospatiales des acteurs par le biais d'une activité participative d'échange visuel et verbal entre l'enquêteur et les participants. Les ateliers étaient organisés de la manière suivante : une première partie était vouée à la discussion collective autour d'une thématique particulière (e.g., le harcèlement de rue), une deuxième partie à la formation photographique de base, et une troisième à la pratique photographique en ville. Dans ce cadre, nous réalisions des prises de vue dans des espaces du quartier préalablement choisis par les participants. Nous nous sommes ensuite employés à la réalisation d'une " cartographie visuelle du genre » $(\mathrm{CVG})$, un exercice qui prévoyait la sélection collective des images (prises lors de la pratique),

8. Cf. Pink, 2013.

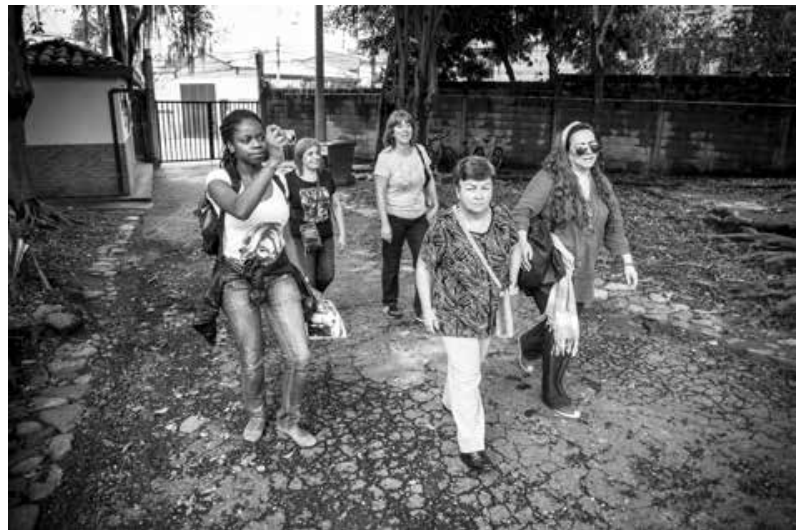

Pratique photographique dans le quartier El Poblado, Medellín.

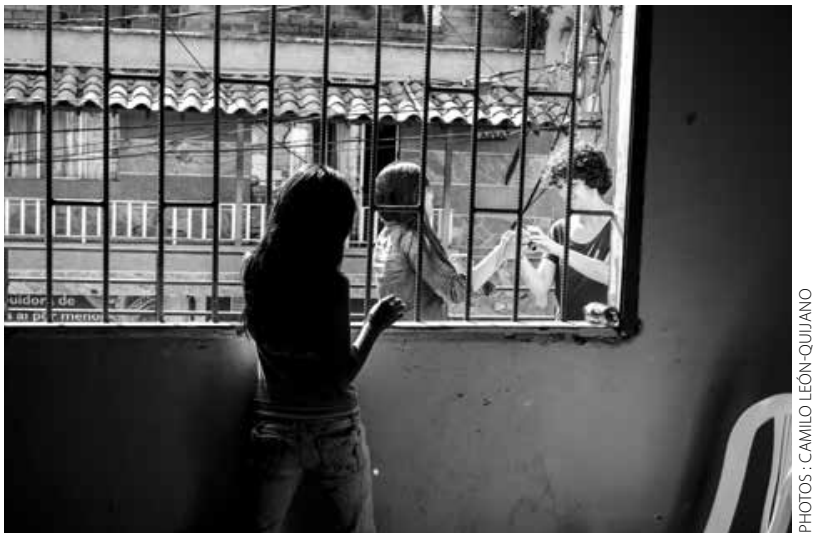

Prise photographique à Comuna 1, La Francia. Thème : le sentiment d'enfermement.

la rédaction de " commentaires descriptifs » (textes ou dessins) sur un grand carton sur lequel les images étaient collées, la socialisation et l'évaluation collective des cartes par groupes de deux ou trois personnes. Finalement, les cartes et les photographies ont été montrées publiquement dans une galerie (Fundación Casa Tres Patios) et lors d'une exposition dans les rues de Medellín (Museo de la Calle).

\section{Transgression de "l'inattention civile " : le contrôle indirect des espaces urbains}

Dans les espaces publics, l'inattention civile permet aux acteurs de vivre dans l'anonymat, avec un engagement minimal tout en "préservant la face » (Goffman, 1963 , p. 84). La transgression de l'inattention civile à Medellín joue un rôle fondamental dans la construction des hiérarchies sociospatiales genrées. Les ateliers photoethnographiques ont été l'occasion de saisir ce type de transgressions «sur le vif ». Lors d'une pratique photographique avec huit femmes de la Comuna 16 (Red de mujeres de Belén), nous avons eu l'occasion d'être témoin des «sanctions indirectes » réservées par les hommes aux 
femmes qui participaient à l'atelier photographique dans le Parque de Belén, un square situé à l'ouest de Medellín?

Les participantes ont attiré les regards et les commentaires des hommes « habitués » du square. Durant l'atelier, elles ont été l'objet de sifflements, de regards et de piropos à plusieurs reprises (notamment quand elles faisaient des photos individuellement $)^{10}$. En discutant ultérieurement de l'expérience sociospatiale lors de la pratique et plus largement de leur expérience en tant que femmes, la plupart des participantes ont avoué avoir une aversion pour cet espace fréquenté majoritairement par des hommes âgés. En analysant leur vécu sensoriel, elles ont affirmé s'être senties «salies » (morboseadas) durant l'atelier, notamment à cause des « regards pervers » (miradas morbosas) jetés par ces hommes. Voici un extrait de la discussion engagée au moment de la socialisation de l'image suivante :

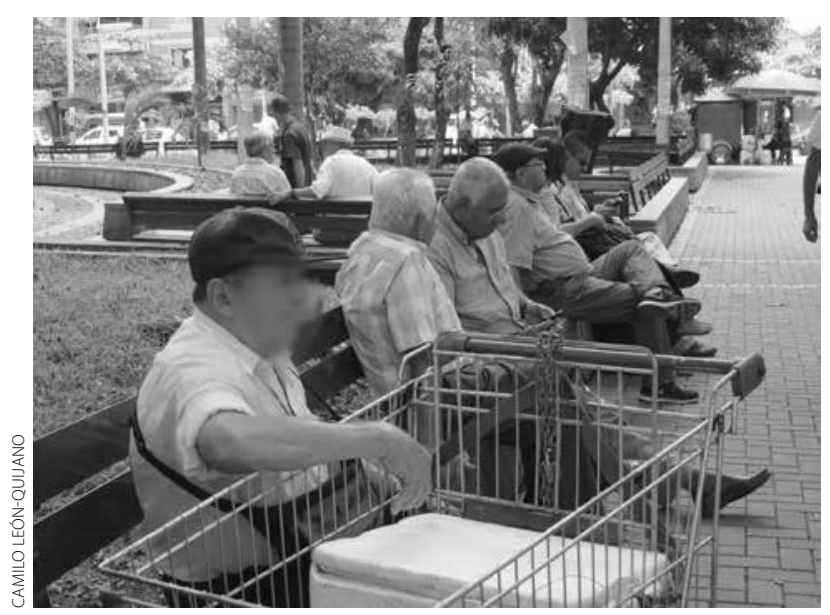

Image prise par une participante au Parque Belén lors de la pratique photographique.

Sandra : [...] Il y a beaucoup d'hommes et nous, en tant que femmes, on se sent très exposées. Celui-ci est un lieu très gênant parce que toujours ils nous font des commentaires très sales... pour cette raison nous avons dessiné cet espace en couleur rouge (sur la CVG).

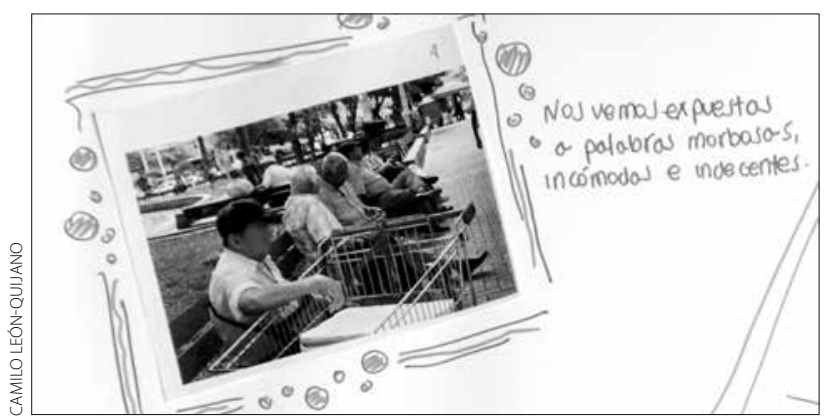

Extrait d'une cartographie visuelle de genre faite au quartier Belén.

9. Square avec une population très majoritairement masculine. Lors de l'atelier, plus de $90 \%$ des individus dans le square étaient des hommes pour la plupart âgés.

10. Pouvant aller de simples sifflements à des commentaires plus directs, explicites et agressifs.

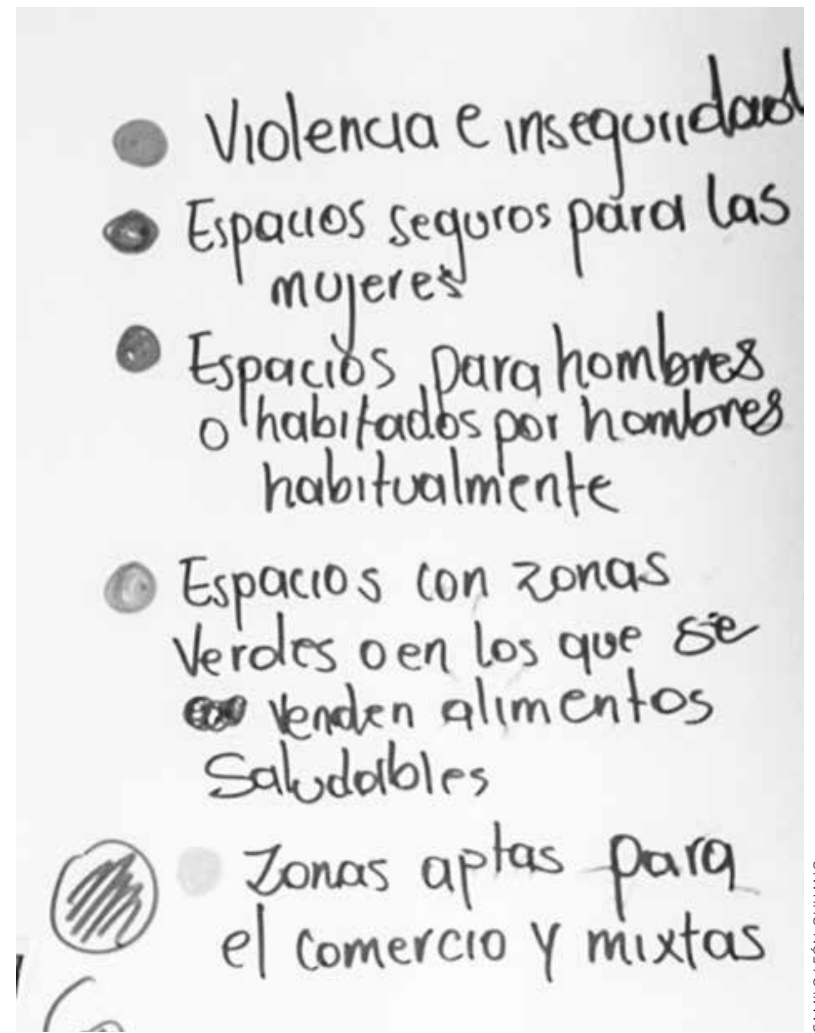

«Code couleur » utilisé pour mettre en lien l'expérience sensorielle avec l'image en question.

L'exercice de cartographie nous a permis d'analyser l'expérience sensorielle des participantes. Au moyen d'une image mettant en perspective le type de population présent dans cet espace, certains témoignages ont aidé à comprendre le malaise ressenti par les participantes qui fréquentent cet espace quotidiennement. À partir de petits gestes quotidiens - sifflements ou «piropos morbosos »les hommes âgés s'approprient indirectement un espace censé être ouvert à l'ensemble de la population.

Dans l'exercice de CVG, les participantes ont choisi d'encercler l'image avec deux couleurs. Le rouge fait référence à la violence et à l'insécurité vécues par les femmes fréquentant le square. Le bleu dénote les espaces habités «par et pour les hommes». En effet, le message iconique de la photographie (Joly, 2009) montre un espace presque exclusivement approprié par des hommes âgés. Pour exprimer le danger et le malaise qu'elles éprouvent dans ce type d'espace, elles ont choisi cette image illustrant le type de rapport sociospatial qu'elles entretiennent dans ce square.

Pour les participantes, ce square est "maluco » (désagréable) et gênant. La présence des femmes est sanctionnée de manière informelle par les piropos et les « sales regards » : "Quand tu marches ils te regardent d'une façon tellement sale que tu te sens dénudée ${ }^{11}$. » À partir de petits gestes quotidiens (regards, sifflements, piropos)

11. «Tu pasas y te lanzan esas miradas que te dejan desnuda! » 
le genre agit de manière «silencieuse » par le biais de la transgression de «l'inattention civile».

La photographie a été un moyen pour « éliciter » des informations liées à cet espace et l'exercice de CVG un moyen pour réfléchir aux multiples contraintes interactionnelles « passives » ou « informelles » subies par les participantes dans cet espace.

\section{Modalisation du corps et présentation de soi dans l'espace urbain}

Valentina habite depuis quinze ans à Medellín. Elle est originaire de Carrillo, un village situé au nord-ouest de Medellín près de la côte Atlantique, où sa famille vit encore. La photographie suivante a été prise sur une plage située près de Carrillo. C'est un lieu paisible de rencontre et de sociabilité entre les villageois. L'image a comme sujet principal deux jeunes femmes, de quinze et vingt ans, présentes au milieu de la plage. Le décor est constitué d'arbres, d'individus au bord de la plage et de quelques débris. C'est une image cadrée avec un plan général et un point de vue à niveau : Valentina situe les deux femmes au milieu du cadre et par sa composition centrale, les deux sujets sont fortement mis en valeur. Nous voyons la figure détendue de la femme qui se trouve à gauche et la figure paisible de sa compagne à droite. Elles portent des vêtements très simples : des t-shirts, des bermudas et quelques bijoux. Elles sont pieds nus et ont des traces de boue sur les jambes. La centralité des sujets, la prise à niveau, le plan général renforcent la figure paisible et détendue des deux jeunes femmes.

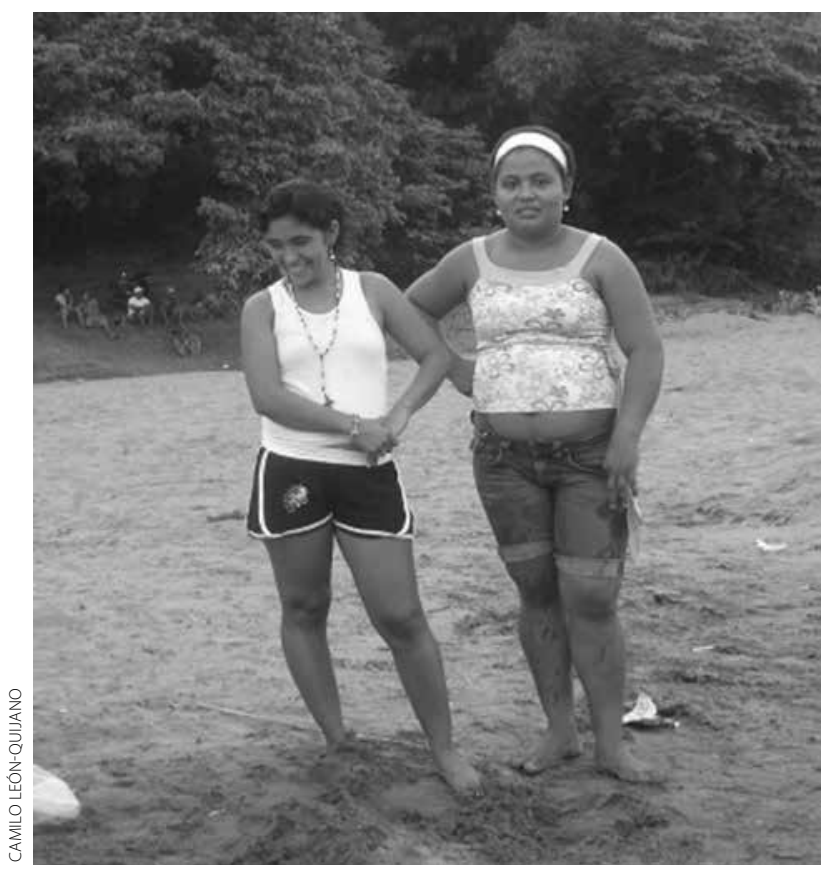

Image de deux jeunes femmes à Carrillo (photographie prise par Valentina, participante aux ateliers).
Cette image a été choisie par Valentina dans une interview photographique pour comparer les pratiques sociospatiales à la ville et à la campagne. Pour elle, l'espace urbain impose des normes corporelles et vestimentaires très strictes : elle ne sort pas de la maison sans être «bien habillée ", sans défriser ses cheveux et sans être maquillée. Au contraire, quand elle rentre chez elle à la campagne, elle s'habille de manière très similaire à ces deux jeunes femmes. Elle ne se défrise pas les cheveux, elle ne sent pas le besoin de se maquiller car elle se sent « moins jugée » à Carillo qu’à Medellín :

Valentina : J'ai voulu prendre la photo de ces deux filles parce qu'elles symbolisent la simplicité, la tranquillité dans laquelle elles vivent. Regarde comme elles sont habillées, elles sont heureuses, elles sont bien. Ici dans la ville non! Comment vas-tu sortir dans la rue comme ça? Camilo : Qu'est-ce qu'il se passe si tu sors comme ça?

Valentina : Peut être c'est un stéréotype, mais les gens vont te dire «Qu'est-ce que t'es mal habillée, t'es si désordonnée... »Par contre, à Carrillo, les gens sont comme ça, ils sont dans la rue et ils ne vont pas te dire que t'es mal habillée, que tu ne te coiffes pas... En fait quand je vais sur la côte [Carillo] je ne me maquille pas, si j'ai la flemme je ne me défrise pas les cheveux parce que mes cheveux sont frisés et quand je suis là ils refrisent. Ici [à Medellín] je ne suis pas capable de sortir avec les cheveux frisés.

La photo des deux jeunes femmes à Carrillo a été l'occasion pour Valentina de raconter son rapport aux deux espaces : la ville et le village. Les normes sociales s'appliquent de manière très différente et surtout la présentation de soi change radicalement dans chaque lieu. À Carrillo, elle peut construire son apparence personnelle de manière " plus libre » qu'à Medellín. Le poids des sanctions directes (ou indirectes) liées à la norme vestimentaire est beaucoup moins important au village qu’à la ville. La réflexion portée par Valentina nous ramène au paradoxe relevé par Gardner (1995, p. 22) : d'un côté les femmes doivent constamment montrer leur appartenance à la classe sexuelle «femme », de l'autre elles doivent faire attention à ne pas transgresser la norme de "vulgarité », faute pour laquelle elles peuvent être «sanctionnées » directement ou indirectement par le pouvoir mâle. Le travail d'elicitation rend compte de cette ambiguité : d'un côté nous trouvons un assouplissement des normes dans des contextes sociospatiaux moins contraignants (le village, Carrillo), de l'autre le poids de normes qui façonnent les pratiques et les «façades personnelles » des femmes - pour prouver qu'elles sont des «vraies femmes ", au sens de Gardner - est plus présent dans les univers urbains de Medellín que dans celui de Carrillo.

\section{« Faire sens » de l'expérience genrée via la pratique photo-ethnographique : les transports et les espaces verts}

Le genre comme " activité consistant à gérer des cours d'actions situées à la lumière des conceptions normatives des attitudes et des activités appropriées à la catégorie de 
sexe à laquelle on appartient » (West et Zimmerman, 2009), permet d'analyser la construction des hiérarchies entre les sexes dans l'espace urbain. Les pratiques photographiques et plus largement l'activité visant à "faire sens » de l'expérience des acteurs (les CVG notamment) ont été un moyen pour éliciter des informations sociospatiales, et plus largement, pour réfléchir collectivement aux modes de construction des pratiques genrées dans la ville. À l'aide de l'image, nous avons analysé le système d'identification et plus particulièrement les pratiques de positionnement des acteurs dans l'espace urbain : « Je me réfère à notre système d'identification, qui implique deux questions liées, nos moyens de découvrir "qui" est celui qui se présente à nous, c'est-à-dire, nos pratiques de positionnement, et nos moyens d'étiquetage (labeling) de ce que nous avons positionné »(Goffman, 1977, p. 86).

L'ethnographie visuelle permet d'établir les modalités d'identification et de positionnement des acteurs sur le terrain en déconstruisant l'opération de prise photographique. Le cadrage, le choix du sujet, de l'objectif, de l'exposition, de point de vue, de plan, supposent la gestation d'une opération cognitive visant à représenter quelque chose. Dans cet esprit, la hiérarchisation visuelle des pratiques spatiales passe par la « re-présentation » sociale de l'espace situé à l'intérieur du cadre photographique.

Afin d'analyser les pratiques genrées des acteurs dans ces espaces, nous utilisons des cartographies visuelles de genre et des discussion menées lors des ateliers pour rendre compte des perceptions sociospatiales des acteurs dans la ville. Les ateliers photo-ethnographiques ont été ainsi un moyen de « faire sens » de l'expérience genrée en confrontant les points de vue des participant-e-s. Un des moments les plus avantageux a été la socialisation et la mise en perspective des cartographies visuelles de genre réalisées dans les différentes comunas.

\section{Pratiques sociospatiales genrées dans les espaces de transports}

Lors d'une exposition collective à la galerie de $\mathrm{C} 3 \mathrm{P}^{12}$ nous avons réussi à confronter les points de vue de plusieurs participant-e-s en discutant de leurs expériences sociospatiales au moment des ateliers. La discussion autour des « espaces de transports » a été particulièrement nourrie et nous a permis de confronter le point de vue d'un participant, Gabriel (trente ans, gardien d'immeuble dans un quartier de classe moyenne à Medellín) à celui d'autres participantes autour des questions de précarité

12. Une exposition finale des CVG a été montée à la galeriefondation Casa Tres Patios (C3P) à laquelle les participant-e-s assistaient pour parler de leur expérience photographique à Medellín. L'idée était de confronter les points de vue des acteurs dans les différentes comunas tout en faisant un travail d'exposition des œuvres et des images faites par les participant-e-s. dans les transports publics et de la pénurie d'offre de transports.

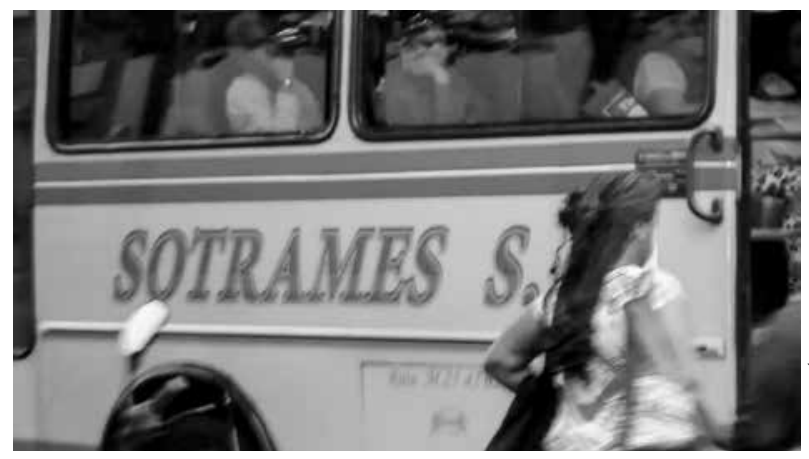

Photographie du bus prise par Gabriel à El Poblado.

Gabriel : Cette photo est le portrait quotidien de la plupart des gens qui n'ont pas les ressources pour s'acheter une voiture... On doit tous utiliser ce moyen de transport, ils nous traitent comme du "bétail » [ganado], il n'y a pas de culture, ils malmènent le passager. J'écris [dans la $C V G]$ « résignation, vie dure », parce que... on doit se battre dans les transports... Je ne fais pas la différence entre les hommes et les femmes, parce que ce qui arrive aux femmes arrive aussi aux hommes... On doit se battre, aller dans les bus et [pour certains] exercer le commerce informel... La femme sur la photo... elle travaille ${ }^{13}$, il n'y a pas de différence... les femmes participent à cette activité informelle. Par conséquent, j'ai de l'admiration envers les femmes, parce qu'elles sont dans la recherche de ressources... mais pour moi en réalité il n'y a pas de différence entre hommes et femmes, on est tous une même unité..

Gabriel dénonce les problèmes quotidiens des usagers du bus : l'inconfort, la contamination et le traitement « bestial » dans les transports publics. En outre, le bus serait un espace réservé aux individus qui n'ont pas les moyens d'acquérir une voiture. En parlant de la relation entre les hommes et les femmes, il évoque l'activité du rebusque ${ }^{14}$ : les femmes et les hommes doivent de la même manière rebuscar (chercher) dans l'informalité des ressources pour survivre dans un contexte de précarité. L'expérience sociospatiale dans le bus serait la même pour les hommes et pour les femmes. Le bus est un espace où les deux sexes " forment une même unité ».

L'image choisie par Gabriel évoque les enjeux présents dans son discours. Au niveau plastique, la photographie est légèrement floue (par le déplacement du bus et des sujets), cet effet transmet un sentiment de mouvement. Le choix du plan général, les lignes diagonales, le point de vue à niveau et le contraste chromatique accentuent le sentiment de dynamisme. Au niveau iconique, nous retrouvons un bus (avec un message linguistique qui parle

\section{L'image représente une vendeuse informelle.}

14. C'est une expression colombienne qui dénote le travail informel réalisé dans le cadre de la survie : rebuscar signifie « chercher partout »; cela passe surtout par les travaux informels comme la vente d'objets variés (alimentaires ou pas) dans les transports. 
du type de transport en question ${ }^{15}$ ), une femme en mouvement et des passagers qui regardent avec « résignation » (resignación) par la fenêtre. La femme porte des sacs et elle a l'air d'être pressée.

À la fin de la séance, Luz (femme de cinquante-cinq ans, fonctionnaire, habitant à la comuna 10) et Elaida (quarante ans, habitant à la comuna 4), participantes à deux autres ateliers, ont exprimé leur point de vue sur cette image :

Luz : Par rapport à l'image du bus et au fait que Gabriel dit que c'est la même chose pour les hommes et les femmes, nous, dans notre atelier on a eu cette même discussion [avec des résultats différents]... Par exemple, moi je dois prendre les bus à Picacho et passer le tourniquet à l'entrée du bus, il m'arrive jusqu'ici [elle fait un geste et met ses mains sur son cou]... Pour nous les femmes c'est plus difficile... tandis que vous [les hommes], vous vous jetez du bus, même s'il est en train de bouger. Par contre, nous on n'est pas capables de faire ça parce qu'on a les enfants, on a les sacs...

Elaida : Pour passer à travers les tourniquets il faut être maigre, ne pas avoir des sacs, et avec les enfants il faut les soulever. On n'a pas la même physionomie et ce sont les femmes qui sont en charge des enfants, qui portent les sacs et on n'a pas toutes la même corpulence. C'est horrible...

Pour Luz, le corps joue un rôle important dans les transports. Elle met en évidence ses difficultés pour accéder aux bus (du fait qu'elle n'est pas très grande) et exprime de même l'image qu'elle a des hommes qui voyagent en bus (« ils peuvent se jeter du bus, même s'il est en mouvement »). Ainsi les hommes et les femmes n'auraient pas la même expérience des transports en commun, du fait de contraintes matérielles (enfants, sacs) ou corporelles modifiant leur rapport à cet espace. Luz et Elaida opposent aux pratiques de positionnement de Gabriel leurs expériences et leurs pratiques de positionnement en tant que femmes. La discussion autour de cette image a permis de confronter leur expérience à la vision «neutre » de l'expérience sociospatiale soutenue par Gabriel.

Dans un autre atelier, nous avons eu l'occasion de discuter autour de l'image suivante (prise au cours d'une pratique photographique par une participante dans le quartier de San Bernardo).

Les huit participantes à l'atelier dans le quartier Belén ont une vision négative des taxis, synonymes d'insécurité. Les participantes ont souvent vu ou vécu de « mauvaises expériences » dans ce type de transport : des violences sexuelles ou des tentatives d'agression sexuelle, des «sales regards " et des commentaires abusifs de la part des chauffeurs :

María P. : J'ai peur, très peur des taxis, d'abord parce que j'ai eu connaissance d'amies qui ont été volées et violées... À plusieurs reprises j'ai dû descendre [des taxis] par peur, parce que moi j'ai très peur dans le taxi, surtout s'il ne suit pas la route [préétablie], parce qu'on n'est pas libre, on est enfermée..

15. Transport de bus privé.

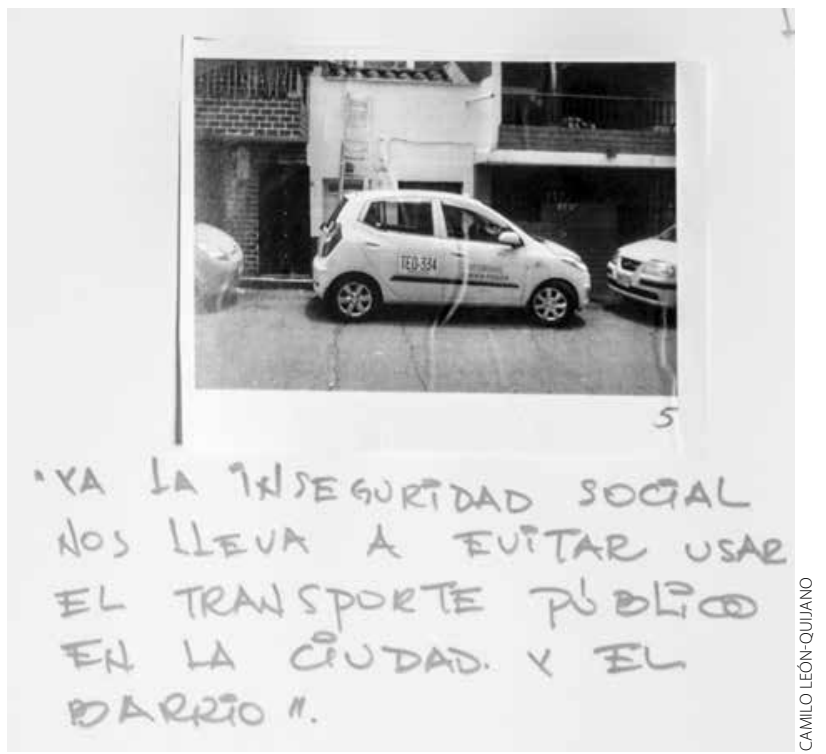

Extrait d'une cartographie visuelle de genre réalisée lors d'un atelier au quartier Belén.

Carolina : Au Mirador de los Alpes, près de l'université de Medellín, il y a une gare de taxis, et passer par là-bas c'est terrible... Ils disent des choses sales, dégoûtantes, c'est pas bien du tout, parfois ils se rapprochent de nous... c'est désagréable...

Eden : On peut tous appliquer [cette technique de détournement du risque], même les hommes parce qu'eux aussi, ils sont aussi vulnérables, ils peuvent être volés. Par exemple, si je vais chez ma mère qui habite à la Frontera... je dis au chauffeur : «Monsieur, vous me dites la plaque d'immatriculation... », j'appelle ma mère ou l'un de mes fils et je leur dis : « Je suis dans le taxi de cette compagnie, je vais à cet endroit. » Je vous donne ce conseil comme un « tip de sécurité »... II faut informer... parce que si ce taxi sait qu'on a informé quelqu'un, cela empêche les mauvaises intentions..

Des formes de contrôle direct (la violence physique/ sexuelle) et de contrôle indirect (piropos, commentaires morbides) se mettent en place chaque fois que Carolina traverse la gare. Ce type d'expérience transforme ses pratiques sociospatiales. Pour María P. et pour Eden, la sensation de «non-liberté » associée à l'absence d'une « zone de sécurité » à l'intérieur du taxi, les amène à adopter des stratégies d'évitement et d'autodéfense (Gordon et Rieger, 1989) : les taxis sont toujours sollicités par téléphone ou par Easytaxi ${ }^{16}$, elles ne prennent pas le taxi dans la rue. En outre, elles développent des stratégies de détournement pour limiter les risques en cherchant un apparent escort (Gardner, 1995, p. 47). L'appel passé par Eden pour avertir le chauffeur que quelqu'un l'attend est une stratégie pour limiter le risque d'agression physique et/ou sexuelle (tout en utilisant ce type de transport).

16. Une application virtuelle pour commander un service de taxi depuis un smartphone. 


\section{Pratiques sociospatiales genrées dans les espaces verts}

Par « espace vert », nous désignons tout espace urbain ou périurbain végétalisé, planté de fleurs ou d'arbres. Souvent le terme est employé par les participant-e-s aux ateliers pour désigner les squares végétalisés, les parcs, les pelouses, les jardins, les bois, les canalizaciones ${ }^{17}$. Les extraits de CVG montrent le " rapport conflictuel » des habitant-e-s aux espaces verts. Dix-neuf des vingt photographies d'espaces verts présentent un « commentaire descriptif ${ }^{18}$ » qui évoque le bonheur, la liberté, la tranquillité, la paix. En même temps, sur sept images, des commentaires évoquent « le danger, l'insécurité, la police ». Ce premier aperçu « conflictuel » des représentations sociales des espaces verts a été constaté lors des discussions que nous avons eues autour de ces espaces. Dans les CVG, côté « bonheur et tranquillité », les espaces verts ont été catégorisés de manière unanime comme des lieux favorisant la détente et «le bonheur » dans la ville: " espaces de détente ", " poumons de notre ville ", « lieux pour profiter ", « nostalgie ", « bonheur ", " calme ». Cependant, côté « danger », ils ont aussi été qualifiés comme « des lieux où il faut faire attention », mêlant « danger », « insécurité », « peur », « crainte d'agression ».

Nous trouvons un cas de conflictualité dans la pratique sociospatiale des acteurs lorsque l'on analyse les témoignages autour des canalizaciones. Ils sont perçus comme des lieux censés promouvoir la réunion des habitants autour d'un espace vert proche de leur domicile, mais aussi comme des lieux peu fréquentés à cause du manque de visibilité (d'éclairage) et de la présence de végétation abondante.

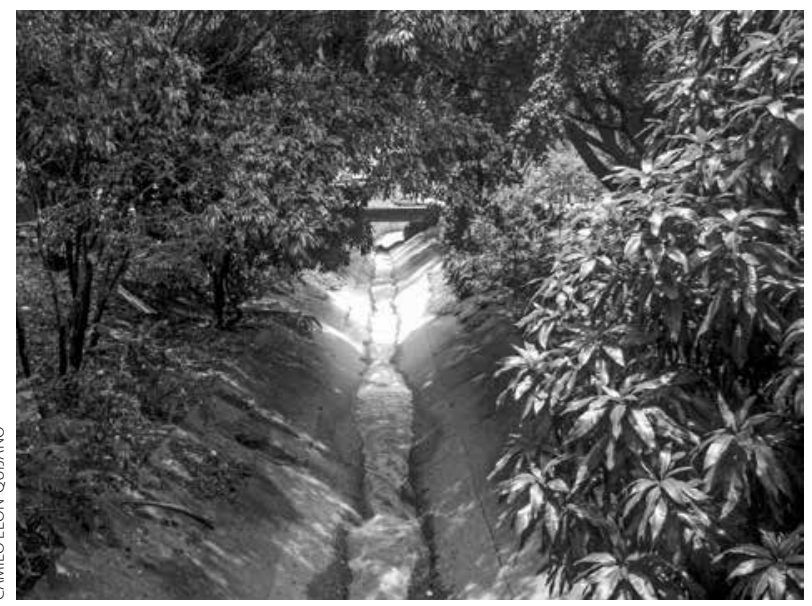

Canalisation près du quartier San Bernardo, comuna 16 (photographie prise par une participante).

17. Une canalización est une structure faite pour canaliser les courants d'eau qui traversent la ville. Normalement elles sont « décorées » avec des plantes. De ce fait, parfois ce sont aussi des lieux sombres et peu fréquentés.

18. Soit par des indications verbales, par des dessins ou par des « codes couleur » qui assignent une couleur à un sentiment.
La photographie, prise légèrement en plongée, montre une canalización entourée de végétation. Au milieu du cadre passe un canal où se reflètent les espaces d'ombre et de lumière. Le plan d'ensemble, le cadrage centré sur une ligne de force centrale (le cours d'eau) et le contraste entre les zones éclairées et non éclairées mettent en évidence les facteurs qui font de cet espace un « lieu dangereux ». Les lieux sans éclairage sont perçus comme des espaces à risque. Lors de la discussion collective, plusieurs participantes ont avoué une appréhension particulière envers les canalizaciones à cause de la faible luminosité. La présence de lumière s'avère indispensable pour circuler tranquillement dans la ville.

Les participants hommes au processus de création de cartographies considèrent ce lieu comme dangereux car ils n'y sont pas à l'abri de violences physiques ou de vols. Les participantes femmes estiment qu'elles pourraient subir notamment des agressions sexuelles directes (viol, attouchement, violence physique) ou indirectes (piropos, sifflements, " regards sales », harcèlement), du fait du manque de visibilité et de l'isolement de cet espace. Par conséquent la fréquentation des espaces verts est extrêmement réduite, tant du côté des femmes que des hommes. Si les femmes et les hommes sont susceptibles d'être victimes de deux types de violence distincts, la pratique sociospatiale des femmes et des hommes est similaire (évitement de ce type de lieu).

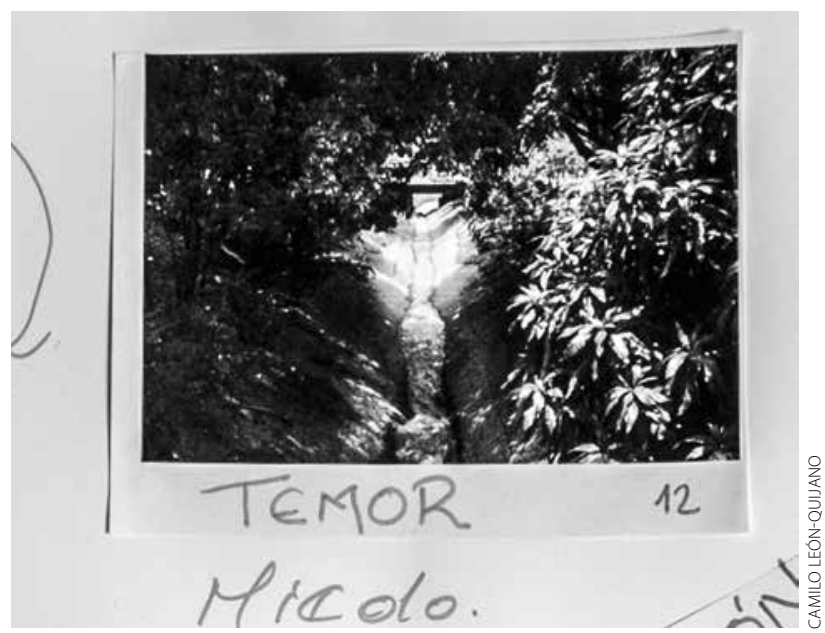

Extrait d'une cartographie visuelle de genre faite au quartier Belén.

La nuit et plus largement le manque d'éclairage public façonnent les pratiques urbaines des femmes ${ }^{19}$, et dans une moindre mesure, des hommes. De la même façon, des espaces plus fréquentés sont perçus comme moins dangereux, tandis que les espaces peu fréquentés sont souvent associés au danger.

Eden (cinquante ans, cadre d'entreprise) : Moi j'ose aller au parc parce qu'il y a un CAI [Centro de Atención Inmediata, un petit centre de police],

19. Cf. Falú, 2009 ; Lieber, 2008. 
parce que je suis courageuse, parce que j'ai mon chien qui me donne confiance et du coup j'y vais tranquillement. Je n'ai pas peur de voir des jeunes qui fument de la marijuana. Mais sans Rocky [le chien] ou sans le CAl je ne sors pas.

Camilo : C'est Rocky qui te donne confiance?

Eden : Ce qui me donne la sécurité, c'est tout d'abord Dieu, Dieu en premier, puis Rocky et le CAI de la police.

Dans le même sens, Bárbara (quarante-huit ans, habitante du quartier) affirme qu'" en tant que femme, on se sent libre dans les parcs si on est protégé par un système de vigilance ». La liberté dans ce type d'espace est associée à un cadre sécuritaire particulier (la présence d'une vigilance privée, de la police, d'un chien). Ainsi, les pratiques genrées y sont souvent influencées par des éléments concrètement repérables (e.g., le CAI, vigilance privée) et des éléments plus « subjectifs » (e.g., Dieu).

L'espace de la ville est un espace social genré, conséquence de représentations, de pratiques, de formes d'appropriation et de contrôle sociospatial hiérarchiques. L'espace, en tant que produit social, façonne les pratiques urbaines des acteurs au moyen de dispositifs de contrôle social formels (la loi, la violence) et informels (ne pas perdre la face et celle d'autrui, réputation, morale) (Goffman, 1977, pp. 109-115). Les méthodes participatives de l'image nous ont permis d'analyser les modalités de contrôle formel et informel des pratiques genrées en confrontant l'expérience urbaine des différentes enquêtées ${ }^{20}$.

Les femmes et les hommes n'ont pas la même expérience de l'espace. Des contraintes et des formes de contrôle formels et informels se mettent en place pour orienter et contrôler les pratiques sociospatiales des femmes dans certains lieux de la ville. Dans cet article nous nous sommes intéressés principalement aux modalités de contrôle informel (regards, sifflements, piropos,

20. Dans ce sens, nous rejoignons les positions de Marylène Lieber (2008) et plus largement de certains géographes, qui ont traité la question du « genre urbain » (Coutras, 1989, 1996, 2002 ; Di Méo, 2011, 2012 ; Raibaud, 2015) : les femmes connaissent des restrictions genrées dans l'usage des espaces urbains par le biais d'agressions et de violences formelles et informelles dans de nombreux espaces de la ville. harcèlement de rue) des pratiques urbaines des acteurs, notamment des femmes. Or, la violence reste le principal mécanisme de contrôle genré des pratiques urbaines des acteurs. Les modalités de contrôle indirect de certains espaces (verts et de transports) se caractérisent par la transgression genrée de l'inattention civile et par le rapport conflictuel des habitant-e-s à certains espaces boisés ou mal éclairés.

En utilisant la photographie comme un outil d'exploration des pratiques sociospatiales, nous avons eu l'occasion d'interroger les modalités de construction du genre en ville. En donnant la possibilité aux participants d'analyser leur propre expérience sociospatiale via l'image, nous avons pu confronter les pratiques genrées des enquêtés. Les pratiques photographiques ont été un moyen d'expérimenter «sur le vif » les modalités de construction du genre en analysant les interactions des participantes dans un espace comme le Parque Belén. De même, les cartographies visuelles ont permis de confronter les expériences genrées des participants en « élicitant » des informations relatives aux pratiques individuelles des femmes et des hommes, notamment dans les espaces de transport. Enfin, la photographie a aussi été un moyen de réfléchir aux pratiques de positionnement et aux modalités genrées de construction des « façades personnelles » des enquêtés.

En définitive, le développement des méthodes participatives de l'image et plus largement d'une ethnographie visuelle phénoménologique, sensorielle, centrée sur les pratiques genrées des enquêtés (McIntyre, 2008 ; Pink, 2011, 2013; Wang et Burris, 1997 ; Woodley-Baker, 2009) contribue à l'étude des modalités de construction du genre dans la ville. Dans cet esprit, la photographie comme moyen de faire sens de l'expérience constitue un outil précieux pour comprendre le rapport genré des acteurs à l'espace.

\section{Remerciements}

Je remercie Juliette Rennes pour sa relecture et ses précieux commentaires. Les idées ici exprimées n'engagent que l'auteur. 


\section{Références bibliographiques}

Bereni L., Chauvin S., Jaunait A., Revillard A., (2012), Introduction aux études sur le genre, Bruxelles, De Boeck.

Collier J., Collier M., (1986), Visual Anthropology: Photography As a Research Method, Albuquerque, University of New Mexico Press.

Coutras J., (1996), Crise urbaine et espaces sexués, Paris, Armand Colin.

Di Méo G., (2011), Les murs invisibles : femmes, genre et géographie sociale, Paris, Armand Colin.

Di Méo G., (2012), «Femmes, sexe, genre : quelle approche géographique? », Espaces et sociétés, 150 (2), p. 149

Falú A., (2009), «Violencias y discriminaciones en las ciudades», in A. Falú (éd.), Mujeres en la ciudad: de violencias y derechos, Santiago de Chili, Ediciones Sur.

Gardner C.B., (1995), Passing by: gender and public harassment, Berkeley, University of California Press.

Goffman E., (1963), Behavior in Public Places: Notes on the Social Organization of Gatherings, New York, The Free Press.
Goffman E., (2002 [1977]), L'arrangement des sexes, Paris, La Dispute.

Gordon M., Rieger S., (1989), The Female Fear, New York, The Free Press.

Harper D., (2002), «Talking about pictures: A case for photo elicitation », Visual Studies, 17 (1), pp. 13-26.

Jaspard M. et al., (2001), « Nommer et compter les violences envers les femmes : une première enquête nationale en France », Population et sociétés, 364, pp. 1-4.

Joly M., (2009), Introduction à l'analyse de l'image, Paris, Armand Colin.

Lefebvre H., (1974), La Production de L'Espace, Paris, Anthropos.

Lieber M., (2008), Genre, violences et espaces publics: La vulnérabilité des femmes en question, Paris,

Les Presses de Sciences Po.

Mclntyre A., (2008), Participatory action research, Los Angeles, Sage Publications.
Pink S., (2011), « Amateur photographic practice, collective representation and the constitution of place », Visual Studies, 26 (2), pp. 92-101.

Pink S., (2013), Doing Visual Ethnography, Los Angeles, Sage Publications.

Raibaud Y., (2015), La ville, faite par et pour les hommes: dans l'espace urbain, une mixité en trompe-l'œil, Paris, Belin.

Scott J., (1988), « Genre : une catégorie utile d'analyse historique », Les Cahiers du Grif, 37 (1), pp. 125-153.

Wang C., Burris M.A., (1997), « Photovoice: Concept, methodology, and use for participatory needs assessment », Health education \& behavior, 24 (3), pp. 369-387.

West C., Zimmerman D.H., (2009), «Faire le genre », Nouvelles Questions Féministes, 28 (3), pp. 34-61.

Woodley-Baker R., (2009), « Private and public experience captured: young women capture their everyday lives and dreams through photonarratives », Visual Studies, 24 (1), pp. 19-35.

\section{Biographie}

CAMILO LEÓN-QUIJANO est doctorant

contractuel à l'École des hautes études en sciences sociales (LAHIC/IIAC-CEMS/IMM) et chargé d'enseignement au sein du master Genre, politique et sexualité de l'EHESS. Ses thèmes de recherche portent sur l'usage des matériaux audiovisuels et sensoriels dans l'enquête ethnographique en milieu urbain. II s'intéresse particulièrement aux usages de la photographie dans l'étude de l'expérience urbaine et du genre. II dirige actuellement le volume 6 dans la revue Visual Ethnography Journal (n¹, 2017), intitulé Visual Ethnography. Tools, Archives, and Research Methods. II publiera également prochainement « Gender, Photography and Visual Participatory Methods » dans la revue Visual Anthropology. II préside également l'Association de recherche sur les méthodes visuelles en sciences sociales Last Focus Visual Research Network. Ses travaux de photographe ont été exposés en Italie, en France et en Colombie (www.camilo-leon.com). 